\title{
Adaptive Joint Lossy Source-Channel Coding for Multihop IoT Networks
}

\author{
Imad Ez-zazi $(\mathrm{D}),{ }^{1}$ Mounir Arioua, ${ }^{2}$ and Ahmed El Oualkadi ${ }^{2}$ \\ ${ }^{1}$ National School of Applied Sciences, Sidi Mohamed Ben Abdellah University, Fez BP 72, Morocco \\ ${ }^{2}$ National School of Applied Sciences, Abdelmalek Essaadi University, Tangier BP 1818, Morocco \\ Correspondence should be addressed to Imad Ez-zazi; imad.ezzazi@gmail.com
}

Received 17 August 2019; Accepted 4 April 2020; Published 11 May 2020

Academic Editor: Ghufran Ahmed

Copyright (C) 2020 Imad Ez-zazi et al. This is an open access article distributed under the Creative Commons Attribution License, which permits unrestricted use, distribution, and reproduction in any medium, provided the original work is properly cited.

\begin{abstract}
We consider monitoring applications in multihop wireless sensor networks (WSNs), where nodes rely on limited batteries so that energy efficiency and reliability are of paramount importance. Typically, lossy compression is aimed at saving transmission energy, yet affects the quality of transmitted data over lossy channels. Accordingly, using error correction coding (ECC) along with compression is required to guarantee both energy efficiency and high-fidelity reconstruction. In this paper, we analyze the energy efficiency of the joint use of lossy compression along with ECC, with the twofold objective of extending the network lifetime and assuring reliability. Specifically, we consider an adaptive joint lossy source-channel coding (JLSCC) system, where the energy efficiency and reliability performances depend on both the compression and the coding rates. Therein, we first carry out a performance analysis of JLSCC, considering realistic models of communication and computational energies, when the communication is performed over a Rayleigh fading channel. Then, we evaluate the performance of the JLSCC system compared to lossy compression and ECC systems in both end-to-end and multihop communications. Our results reveal that an adaptive JLSCC results in substantial energy saving while guaranteeing the required reliability performance, compared to both lossy compression and channel coding systems, that cannot be efficient for both energy and reliability. Instead, the JLSCC system is proved to be energy efficient for small distance end-to-end communication and large-scale multihop network, while leading to satisfactory reliability performance.
\end{abstract}

\section{Introduction}

Wireless sensor networks (WSNs) and their progress into the internet of things (IoT) have recently experienced a tremendous upsurge. This recent paradigm has attracted significant attention and extensively found applications in many fields, e.g., smart cities, environment monitoring, health care, and industry [1-3]. In WSN, nodes are mostly deployed in large numbers to cooperatively and efficiently collect and disseminate sensed data on a large scale. In most scenarios, sensor nodes are likely going to be battery-powered and autonomously relying on limited energy supply. However, nodes are expected to be stand-alone and able to run for many years without human intervention as replacing depleted batteries may be a costly and impractical, if not impossible in hostile environment [4]. Thus, designing energy-aware algorithms and protocols in order to prolong the network life- time is the central challenge consideration in WSN and IoT applications.

In monitoring applications, sensor nodes periodically sense the surrounding environment and exchange dependably the sensed information to the base station (BS), whose direct communication would hastily consume the limited batteries [5]. In large-scale WSNs, numerous nodes tend to cooperate effectively and communicate the sensed measurements to the BS, that is, by regularly switching the role of sensing and relying operations. In this case, the sensed data is likely going to be processed and forwarded through various hops before reaching the BS according to suitable routing protocols, in order to reduce the required transmission energy and extend the multihop network lifetime [6]. Moreover, in such networks, the amount of gathered data to be managed by the network is expected to be prohibitive in the one hand and highly correlated in time and space in 
the other hand, due to the fact that nodes are extensively and densely deployed. Accordingly, reducing the size of the transmitted data by applying signal processing techniques is a crucial concern to save transmission energy. This is typically carried out by using lossy data compression [7], in both time and space [8], at source nodes, and data aggregation at relay nodes [9], as the data is routed through the network. Although lossy compression enables to discard more intrinsic data redundancy, it introduces a distortion into the original signal, which is required to be transmitted reliably, and successfully reconstructed at the receiver end [10]. This allows to reduce the required transmitted energy, yet makes the compressed signal more error-prone in a large-scale and lossy environment. In this case, the reconstruction of original data reliably by the decompressor at the BS is a challenging task, as the signal is supposed to cross a wide area meeting various obstacles, interferences, and multipath fading through the network. Consequently, the use of joint lossy source-channel coding (JLSCC) based on lossy compression and error correction coding (ECC) is of paramount importance in multihop WSN to provide both energy saving and reliable data reconstruction at the BS.

ECC approach has been recently deemed to be crucial for both transmission energy saving and reliable communication thanks to their coding gain $[11,12]$. Ideally, coding schemes enable to tackle various channel impairments and data loss by using lower transmission energy compared to uncoded scheme. The main rationale behind compression and ECC techniques is their ability of trading some extra processing energy of compression (i.e., encoding) and channel coding (i.e., decoding) for eminent energy saving in the transmission energy [13]. However, using lossy compression along with ECC raises various tradeoffs in energy-constrained WSN. For instance, using higher compression ratios to save supplementary transmission energy entails dissipating higher processing energy for the compressor. For the ECC case, the strongest coding schemes assure higher coding gain (i.e., transmission energy saving) and improved reliability; however, they require more processing energy consumption for the channel decoder. Toward this end, if the extra processing energy consumed at the compressor and the channel decoder outweighs the transmission energy saving due to compression and coding gains, then neither compression nor ECC coding would be energy efficient, especially in multihop sensor networks where transmission hops are short, which makes the processing energy as important as the transmission energy.

In this paper, we thoroughly investigate and analyze the energy efficiency and energy-reliability tradeoffs when using jointly lossy compression schemes along with ECC techniques in energy-constrained multihop WSN. The integrated energy analysis is based on a framework that considers all energy components of communication (i.e., transmission and reception), processing (i.e., lossy compression and channel decoding), and transceiver circuitry, in order to efficiently assess the energy gains/costs of using the JLSCC system in WSN. Accordingly, we emphasize the impact of both communication and processing costs of JLSCC on communication reliability. The main goal of this paper is to evaluate the energy efficiency and reliability performances when using jointly lossy compression along with ECC schemes in noisy multihop WSN.

The remainder of this paper is organized as follows. Section 2 discusses and analyzes the related works. The system model of the joint lossy source-channel coding including the compression, error control, and channel models is described in Section 3. Section 4 discusses the energy consumption and energy efficiency of the JLSCC system relying on realistic models of communication and computational energies. The simulation results and energy performance analysis of the JLSCC, lossy compression, and ECC are revealed and discussed in Section 5. Section 5.2 concludes the paper.

\section{Related Works}

Over the last decade, a tremendous literature has been dealing with the energy efficiency for IoT and WSN $[14,15]$. In energy-constrained WSN, nodes are either powered by limited batteries, which embody the only source of energy [16], or equipped with energy-harvesting (EH) system to provide energy neutrality [17]. In this work, we consider batterypowered devices without energy-harvesting capabilities and we investigate energy efficiency in multihop WSN. In this context, many works have been devoted to various networking approaches at all layers of the protocol stack such as routing [18, 19], channel access [20, 21], data gathering and dissemination [9], systematic compression and control mechanisms [22, 23], and cross-layer optimization [24]. However, most of these works have been neglecting some critical and paramount concern parameters, such as the effect of channel distortions on energy and reliability performances, the impact of processing energy of both compression and channel coding, and the resulting energy-reliability tradeoffs for battery-powered WSN.

Among prior works, lossy compression has been deemed a pivotal energy-efficient technique for energy-constrained WSN [25]. In this regard, a considerable literature has been emerged on lossy compression for the sake of minimizing energy and prolonging the battery's lifetime [26, 27]. For instance, in [7], the authors have investigated energy saving and computational energy tradeoffs of various temporal lossy compression schemes based on linear approximation and Fourier transforms. It has been proven that Lightweight Temporal Compression (LTC) scheme [28] is the most energy efficient in terms of both compression performance and computational energy. However, these works have neglected the effect of packet loss on the compressed and transmitted readings, by assuming that the data is correctly received by the BS. Moreover, direct communication between sensor nodes and the BS has been the most adopted scenario, yet multihop transmission, where both communication and processing energies as well as the distortions extensively impact the WSN system, has not been considered. In recent works, communication reliability has been considered as important as energy efficiency in WSN and IoT applications [29]. For this reason, providing energy efficiency and reliability is a high demand issue in recent and 
next IoT paradigms [30]. In this context, the joint use of lossy compression and error correction coding is a key approach to deal with the twofold requirements, i.e., energy and reliability. However, few works have been dealing with the JLSCC optimization in WSN [31, 32], limiting their study on single-hop communication systems. In [31], the authors have studied the use of JLSCC in a point-to-point channel with an energy-harvesting transmitter, yet they have centered their work on the distortion performance without considering the energy efficiency. That is by neglecting both the transmission energy saving of JLSCC systems and the effect of processing energy of lossy compression and channel coding systems on node energy. Similarly, in [32], the authors have investigated the energy-neutral source-channel coding system in end-to-end scenario, by optimally allocating the energy oversource acquisition, compression, and transmission. However, computational energy of channel coding and lossy compression and their effect on energy efficiency of battery-powered nodes have not been taken into account. In [33], a source and channel coding for energy-limited wireless sensor node has been examined, where a Gaussian source is transmitted over a flat fading channel and the offline minimization of the total distortion over a finite-time horizon is considered. Therein, the optimal distortion and transmission energy are obtained; yet like many other works [34,35], they limit their studies to Gaussian data sources and the processing energy is neglected. In our previous work [36], an analysis of joint lossy compression and channel coding in end-to-end communication is carried out. This work has investigated the impact of compression and coding rate allocation on the performance of both data reconstruction fidelity and communication reliability. However, the energy performance of JLSCC in constrained sensor nodes has not been considered. Thus, both transmission energy efficiency and effect of the extra computational costs of JLSCC have not been studied. Besides, the presented system has focused on single-hop communications, where data reconstruction and recovery are performed at the BS.

Our work bears similarities with [37], where nodes are equipped with a lossy compression and channel coding systems with energy-harvesting capability, yet we consider battery powered devices. This work has proposed an energy-aware joint source-channel coding approach that minimizes the expected distortion at the receiver, under the constraints imposed by the EH system. Nevertheless, it has supposedly neglected the computational energy of both channel coding and decoding processes, the extra transmission costs of coding redundancy, and the energy saving stemmed from the ECC gain. In addition, they have assumed a direct transmission, where data reconstruction and decoding are carried out at the BS, thus without excessive decoding energy. We note that when transmission takes place over small distances, the processing energy cost of running data acquisition systems (e.g., compression and decoding) may be comparable if not exceeding that of radio transmission [13]. Several previous papers have addressed the tradeoff between the processing energy of error-correcting codes and their transmission energy saving in the one hand and between reliability and total energy consumption in the other hand $[38,39]$. These works have commonly underscored the impact of the computational energy of error correction coding and decoding on the performance of both energy efficiency and reliability in WSN. In this case, performing lossy compression at transmitter nodes and channel decoding at intermediate nodes or cluster heads $(\mathrm{CH})$ could worsen the energy efficiency of sensor nodes, yet this issue has been widely neglected, focusing separately on either compression or channel coding processing. In return, this work is aimed at investigating the energy efficiency of joint lossy sourcechannel coding by considering communication and processing energies for both lossy compression at transmitter and channel decoding at intermediate nodes in a multihop architecture. We notably emphasize the tradeoffs between the computational costs and the energy gains of JLSCC and their impact on energy efficiency. Therein, we study both energy efficiency and distortion performances of the JLSCC system for nodes and for network lifetime and the effect of packet loss and coding on data distortion.

\section{System Model}

We consider a multihop sensor network comprised of $N$ sensor nodes that constantly monitor a phenomena of interest and communicate data cooperatively to a distant BS. Each sensor node in the network is assumed to be batterypowered with a limited energy supply of a size $B$. The communication between source nodes $\left(S_{i}\right)$ and intermediate nodes $\left(R_{i}\right)$ is carried out by a lossy source-channel coding system, where in each communication block, a source sequence of fixed length is mapped into a channel codeword whose length depends on the available battery charge and channel condition. We assume a Temporal-Division Multiple Access (TDMA) for transmission scheduling, where time is partitioned into slots denoted by $t \in(0,1, \cdots)$. Accordingly, at each time slot $t$, nodes decide whether to compress data for source coding, to encode data for channel coding, and finally to convey data to the BS where it will be reconstructed, decoded, and correctly retrieved. Figure 1 describes the system model of a node communication system.

3.1. Lossy Compression. Each node in the network compresses its sensed data using a lossy compression scheme to squeeze the data size and save transmission energy. Nodes compress data blocks by selecting a compression ratio $\rho_{i} \epsilon$ $[0,1]$ and generate a compressed data block of size $L$ given that each block has an initial constant size of $L_{0}$ [36].

Given an original block of sensed data $L_{0}$ and and its compressed version $L$, the compression ratio $\rho$ is defined as the ratio between the size of compressed block and that of the original one:

$$
\rho_{i}^{(t)}=\frac{L_{i}^{(t)}}{L_{i, 0}^{(t)}} .
$$

The compression ratio $(\rho=0)$ indicates that no packet is transmitted $(L=0)$, while $(\rho=1)$ refers to no compression takes place $\left(L=L_{0}\right)$. 


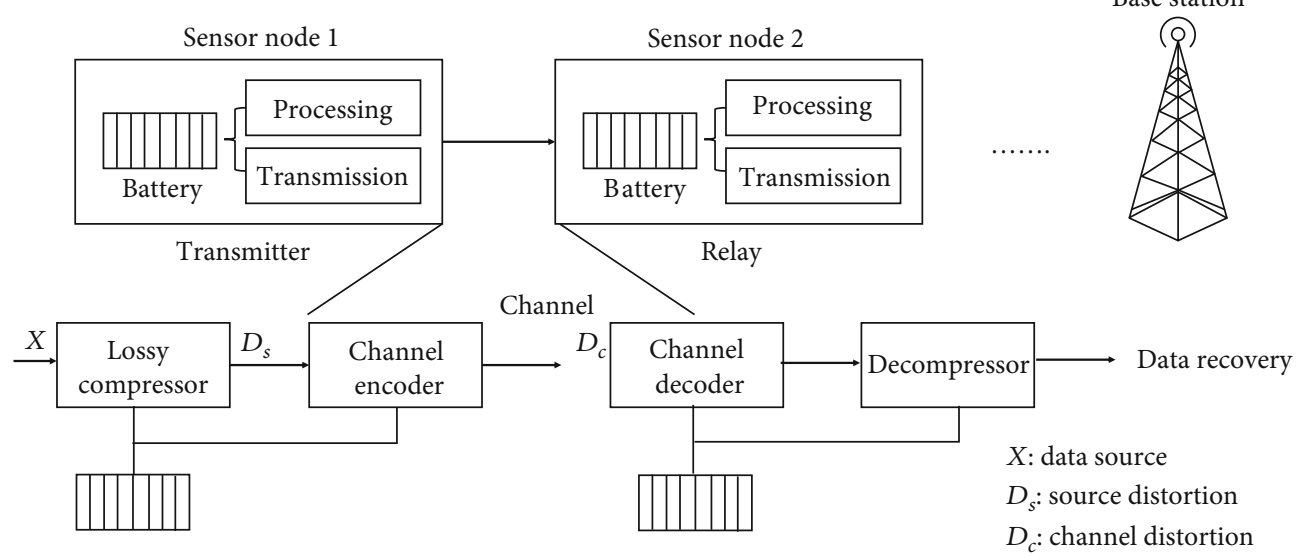

FIGURE 1: Block diagram of sensor nodes: sensors composed of a source encoder, channel encoder, decompressor, and channel decoder, powered by a limited battery.

In this work, we consider the LTC scheme that has been proven to provide excellent performance in terms of energy consumption and distortion tradeoff, which lead to be a reference scheme for energy-constrained sensor networks. LTC is a low complexity algorithm that compresses a time series by creating an approximate representation through line segments according to a predefined error tolerance $\varepsilon>$ 0 . The algorithm consists of approximating multiple readings through a single segment, so that the segment is within the preset error tolerance for all point. LTC transmits the end point of line segments instead of the original data. This temporal lossy scheme yields excellent performance when the signal is highly correlated in time as the case in most WSN application that constantly report data to the BS. Depending on the lossy compression level (i.e., compression ratio) selected by nodes, a distortion $D_{i}(\rho)$ is introduced within the transmitted data which can be mathematically modeled as follows:

$$
D_{i}^{(t)}(\rho)=\left[b_{i}\left(\frac{1}{\left(\rho_{i}^{(t)}\right)^{a_{i}}}-1\right)\right]^{+},
$$

where $b>0,0<a<1$, and $[.]^{+} \cong \max (., 0)$. These parameters have been derived empirically fitting the realistic rate-distortion curves of lossy compression $\left(a_{1}=0.35, b_{i}=\right.$ 19.8) [7].

The distortion is null when the packet is not compressed (i.e., $\rho_{i}^{(t)}=1$ ). However, the distortion increases as much as we compress the data (i.e., decreasing the compression ratio). Although decreasing the compression ratio brings about saving more transmission energy, it increases the distortion that complicate the data reconstruction at the destination, especially in highly noisy environments.

3.2. Error Correction Coding. Depending on the channel stats, the transmission medium introduces a channel distortion $D_{c}$ into the transmitted signal that refers to channel impairments. In this case, the data is received with a total distortion including the source and channel distortions $\left(D_{\text {tot }}=D_{s}+D_{c}\right)$ at both intermediate nodes and the BS, which complicate data reconstruction and retrieving [36]. Consequently, ECC is mandatory for lossy compressed data to guarantee transmission reliability through lossy channels and assure energy efficiency. After compressing the data blocks at the compressor, the channel encoder encodes the data using suitable linear block codes due to their performance and low complexity [40]. ECC has been mainly used to handle the problem of channel impairments and energy saving in WSN [41]. Nodes encode the data blocks by selecting an adequate coding rate $r \in[0,1]$ that decides the quantity of redundancy to be added to the compressed block before transmission and allows the decoder to decode and retrieve the original data. The encoder receives the compressed blocks of size $L$ and produces encoded blocks (i.e., codewords) of size $(n=L+\tau)$, where $\tau$ is the adaptive number of redundancies.

Let $\left(L \leq L_{0}\right)$ be the compressed packet from the lossy source coding and $(n \geq L)$ is the transmitted codeword; the coding rate is defined as the ratio between the size of the compressed block and that of the encoded one:

$$
r_{i}^{(t)}=\frac{L_{i}^{(t)}}{n_{i}^{(t)}},
$$

where $n=(L / r)=\left(\rho L_{0} / r\right)$ is the resulting codeword to be transmitted over the channel at a time slot $t$.

The coding rate of transmission is selected according to the implemented error correction scheme. In this work, we consider the link-layer Reed-Solomon (RS) codes as ECC schemes due to their performance and reasonable decoding complexity [42]. An $R S(L, n)$ channel code with a coding rate $r$ allows to convert every $L$-compressed information symbols into $n$ symbol block by including $(n-L)$ parity symbols. This enables the decoder to correct any combination of errors less than the correction capacity $\omega_{R S}=((n-L) / 2)$. At the receiver ends, $L$-original packets can be reconstructed by receiving any $L$ out of $n$ packets $(n>L)$. 
3.3. Channel Model. We consider a none-selective block Rayleigh fading channel, where the channel gain $H$ keeps constant during a time slot. Accordingly, depending on the selected coding rate $r$ and channel conditions, the packet may not be correctly received with an outage probability $P_{\text {out }}(r)$. In this case, when the channel is in a deep fade, the packet is lost and an outage occurs. Therein, the results of finite-length information theory that adapt the classical concepts of channel capacity to the case of short data packets is considered in this work [43], due to the fact that packets sent by sensor nodes are likely to be short. In particular, the results of [44] legitimate the use of the quantity $\log (1+\gamma)$ to represent the maximum coding rate even in the finite-length regime, where $\gamma$ is the SNR at the receiver, given by

$$
\gamma=\frac{|H|^{2} P_{t x}^{(t)}}{A^{2}\left(d / d_{0}\right)^{\alpha} N}=|H|^{2} \widehat{\gamma}
$$

where $H$ is the channel gain coefficient that represents fading and $\hat{\gamma}$ is the expected SNR at the receiver that depends on the transmission power $P_{t x}$, the noise power spectral density $N$, and the path-loss expression $A^{2}\left(d / d_{0}\right)^{\alpha}$. The path loss is expressed as the path-loss exponent $\alpha$, the distance between transmitter and receiver $d$, and the path-loss coefficient $4 \pi d_{0} f_{0} / c$, where $f_{0}$ is the transmission frequency, $c$ is the speed of light, and $d_{0}$ is a reference distance for the antenna far field [45].

When the channel is in a deep fade (i.e., $|H|$ is small), the packet is lost and a packet erasure (i.e., channel outage) occurs. The corresponding outage probability at the receiver is expressed as

$$
P_{\text {out }}(r)=\operatorname{Pr}\left(\log _{2}(1+\gamma)<r\right) .
$$

The channel gain $H$ follows a complex Gaussian distribution with zero mean and unit variance, as the channel is considered to be Rayleigh fading. Therefore, the outage probability can be defined as

$$
P_{\text {out }}(r)=1-e^{-\left(2^{r}-1\right) / \hat{\gamma}} .
$$

\section{Energy Analysis of JLSCC}

The sensor network lifetime is determined by the lifetime of all deployed node batteries. Note that all nodes in the network are energy neutral such that $B_{i}^{(t)}$ denotes the battery level of the sensor node $i$ at a time slot $t$. In each period of communication, a nonnegative amount of energy $E_{i}^{(t)} \in\left[0, B_{i}^{(t)}\right]$ is consumed by nodes for communication, processing, and circuitry operations. However, we consider that the energy spent in sensing process is negligible with respect to that spent for compression, error correction decoding, and transmission.

To fully assess the total energy consumption at the transmitter and the receiver, all signal processing operations at the transmitter and the receiver are considered in the energy model. The total power consumption at the transmitter and receiver at a time $t$ are, respectively, given by

$$
\left\{\begin{array}{l}
E_{T_{i}}^{(t)}=E_{t x}^{(t)}+E_{c}^{(t)}+E_{P}^{(t)}(T), \\
E_{R_{i}}^{(t)}=E_{c}^{(t)}+E_{P}^{(t)}(R),
\end{array}\right.
$$

where $E_{t x}^{(t)}$ is the transmission energy at a time slot $t$ and $E_{c}^{(t)}$ denotes the static energy consumption of the circuit blocks at the transmitter and receiver. $E_{P}(T)$ and $E_{P}(R)$ are the processing energies (i.e., lossy source-channel coding) at the transmitter and receiver, respectively. The total processing energy at a time slot $t$ is the sum of both processing energies at the transmitter (i.e., compression and encoding) and receiver (i.e., reconstruction and decoding):

$$
E_{P}^{(t)}=E_{P}(T)+E_{P}(R) .
$$

The total energy consumption of a transceiver equipped with a lossy source-channel coding is obtained as the sum of energies consumed at the transmitter $E^{(t)} T_{i}$ and next receiver $E^{(t)}{ }_{R_{i+1}}$, where we refer to $E_{i}^{(t)}$ as the average energy expenditure per time slot.

$$
E_{i}^{(t)}=E^{(t)} T_{i}+E^{(t)}{ }_{R_{i+1}} .
$$

For $N$ nodes distributed in a multihop network, the energy consumed in transmission and transmitter processing is considered for all nodes. However, the energy consumed in receiving and relay processing is counted for all nodes except source nodes. Thus, the total energy of a multihop network over $x$ hops of transmission is given as

$$
E_{\mathrm{tot}}^{(t)}=\sum_{i=1}^{x} E^{(t)} T_{i}+\sum_{i=1}^{x-1} E^{(t)} R_{i+1} .
$$

4.1. Transmission Energy. The transmission energy depends on the compression and coding rates of the lossy source and channel coding, while the reception energy is considered to be relied on the circuitry costs. Thus, the transmission energy cost given a transmit power $P_{t x}$ and period of length $T$ is expressed as

$$
E_{t x}^{(t)}=\frac{T P_{t x}^{(t)}}{\eta_{A}}
$$

where $\eta_{A} \in(0,1]$ is the efficiency of the antenna's power amplifier.

The use of ECC schemes enables to operate at significantly lower Signal to Noise Ratio (SNR) than an uncoded system, for the same Bit Error Rate (BER). This provides sufficient energy savings (i.e., lowered minimum transmitted power requirement) due to the coding gain $\left(C_{\text {gain }=\gamma_{u}-\gamma_{\mathrm{ECC}}}\right)$ acquired by the ECC scheme. For instance, the minimum required transmit power $P_{t x, U}$ for an uncoded system at the 
SNR required to achieve a desired packet error rate (PER) is given as follows:

$$
P_{t x, U}^{(t)}=A^{2}\left(\frac{d}{d_{0}}\right)^{\alpha} N \cdot B 10^{\left(\gamma_{u}^{(t)}+F\right) / 10}
$$

where $\gamma_{u}^{(t)}$ is the required SNR for uncoded block at a time slot $t, B$ is the signal bandwidth, and $F$ is the receiver noise figure.

For a coded system, the minimum required transmit power $P_{t x, \mathrm{ECC}}$ given the required SNR of coded data $\gamma_{\mathrm{ECC}}<$ $\gamma_{U}$ is expressed as

$$
P_{t x, \mathrm{ECC}}^{(t)}=A^{2}\left(\frac{d}{d_{0}}\right)^{\alpha} N \cdot B 10^{\left(\gamma_{\mathrm{ECC}}^{(t)}+F\right) / 10}=\frac{P_{t x, U}^{(t)}}{10^{\left(C_{\mathrm{gain}} / 10\right)}} .
$$

The transmission energy of compressed data blocks at a time slot $t$ depends on the minimum transmission power and compression ratio, which is expressed as

$$
e_{t x}^{(t)}(\rho)=T_{U} \frac{P_{t x, U}^{(t)}}{\eta_{A}}=\rho^{(t)} L_{0} \cdot T_{b} \frac{P_{t x, U}^{(t)}}{\eta_{A}}
$$

where the duration $T_{U}\left(=L \cdot T_{b}=\rho^{t} L_{0} \cdot T_{b}\right)$ defines the number of compressed bits to be transmitted, with $T_{b}$ being the fix bit duration that depends on the adopted modulation scheme.

When the data is compressed and coded with an ECC scheme, the transmission energy is expressed as function of both compression ratio and coding rate, as follows:

$$
\begin{aligned}
\hat{e}_{t x}^{(t)}(\rho, r) & =T_{\mathrm{ECC}} \frac{P_{t x, \mathrm{ECC}}^{(t)}}{\eta_{A}}=\left(\frac{\rho}{r}\right)^{(t)} L_{0} \cdot T_{b} \frac{P_{t x, \mathrm{ECC}}^{(t)}}{\eta_{A}} \\
& =n^{(t)} \cdot T_{b} \frac{P_{t x, \mathrm{ECC}}^{(t)}}{\eta_{A}}
\end{aligned}
$$

where $T_{\text {ECC }}$ is the length of the channel codewords to be transmitted $\left(T_{\mathrm{ECC}}=n \cdot T_{b}=(\rho / r){ }^{(t)} L_{0} \cdot T_{b}\right)$. The codeword of the channel coding scheme $n$ to be transmitted at a time slot $t$ rests on both coding and compression rates. Sensor nodes are supposed to tune both compression ratio and coding rate according to the battery level and channel state. Note that when the compression ratio $(\rho=1)$, the data is transmitted without compression, if the coding rate $(r=1)$, the data is transmitted uncoded, and when $(r, \rho=0)$, the data is not transmitted at the current time slot.

4.2. Computational Energy. The processing energy $E_{\text {proc }}$ dissipated due to the lossy source-channel coding concerns the energies consumed on the compressor, the channel encoder, and the channel decoder as data reconstruction and decompression are supposed to be carried out at the BS. However, the lossy compression and channel decoding are the most energy demanding compared to channel encoding operations.

$$
E_{\text {proc }}=\left(E_{P_{\text {comp }}}+E_{P_{\text {enc }}}+E_{P_{\text {dec }}}\right) .
$$

For both lossy compression and channel coding, we computed the number of additions, multiplications, divisions, and comparisons executed at sensor nodes. Accordingly, these operations are efficiently translated into the corresponding number of clock cycles according to the considered sensor hardware. Therein, the associated energy dissipation is derived, similarly to [46]. In this regard, the MSP430 Microcontroller Unit (MCU) from Texas Instruments is considered, in which the number of clock cycles for each operation is detailed in [47]. Moreover, the energy consumption per clock cycle, $E_{\mathrm{cc}}$, is determined by taking into account the MSP430F1612. This microcontroller family consumes $330 \mu \mathrm{A}$ in active mode when the MCU operates at $1 \mathrm{MHz}$, and the supply voltage is $+2.2 \mathrm{~V}$. Thus, the energy consumption per clock cycle is given as

$$
E_{\mathrm{cc}}=330 \mu \mathrm{A} \times \frac{2.2 \mathrm{~V}}{1 \mathrm{MHz}}=7.26 \cdot 10^{-10} \mathrm{~J} .
$$

4.2.1. Lossy Compression. The energy consumed by the lossy compression algorithms is estimated by mapping the number of arithmetic instructions required to process the signal into the corresponding energy demand:

$$
E_{P_{\text {comp }}}^{(t)}= \begin{cases}E_{\mathrm{cc}} L_{0} N_{\mathrm{cc}}(\rho), & 0<\rho_{i}^{t}<1, \\ 0, & \rho_{i}^{t} \in\{0,1\},\end{cases}
$$

where $E_{\mathrm{cc}}$ is the energy consumption of the microcontroller in one clock cycle, $L_{0}$ is the number of bits to be compressed, and $N_{c c}$ is the number of clock cycles required by the lossy compression algorithm per uncompressed bit. By leveraging on the results of [7], $N_{\mathrm{cc}}$ is expressed as a function of the compression ratio and given as follows:

$$
N_{\mathrm{cc}}(\rho)=\left(\sigma \rho_{i}^{t}+\beta\right),
$$

where $(\sigma, \beta>0)$ are two fitting coefficients.

Note that when at $\rho_{i}^{t}=\{0 / 1\}$, the node does not perform any compression; thus, we have $E_{P_{\text {comp }}}^{(t)}(\rho)=0$. The data reconstruction and decompression are assumed to be carried out at the BS. Consequently, no additional cost of decompression is computed at intermediate nodes.

4.2.2. Error Correction Coding. ECC based on linear block codes have typically simple encoding process with insignificant computational energy that can be tackled by the energy resources of nodes [22]. However, decoding is a complex process which requires significant energy expenditure. In multihop architecture, the decoding energy cost is likely going to be considered repetitively at several intermediate nodes which results in eminent energy consumption and network drain. Accordingly, decoding energy of ECC schemes is required to 
be accounted along with compression cost in the JLSCC system. The computational complexity of ECC decoding grows exponentially by increasing the error correction capability and decoding performance. Leveraging on [48], the decoding latency of linear coding schemes is expressed as a function of the coding length $n$ and correction capacity $t_{c}$ as follows:

$$
T_{\mathrm{dec}}=\left(2 n \cdot t_{c}+2 t_{c}^{2}\right)\left(T_{\mathrm{add}}+T_{\text {mult }}\right),
$$

where $T_{\text {add }}$ and $T_{\text {mult }}$ are the latencies associated with the addition and multiplication operations, respectively. Moreover, a 16-bit MSP430 microcontroller requires 184 and 395 clock cycles to perform additions and multiplications, respectively. Therefore,

$$
T_{\text {add }}+T_{\text {mult }}=579 \cdot\left[\frac{m}{16}\right] T_{\text {cc }}
$$

where $T_{c c}$ indicates the one cycle duration of processor and $m=\left[\log _{2} n+1\right]$, respectively. Based on the decoding latency, the total energy of the decoding process can be calculated as follows:

$$
E_{\mathrm{dec}}=V I_{\mathrm{proc}} T_{\mathrm{dec}}
$$

The decoding energy (Equation (22)) can be reformulated as function of the energy clock cycles $E_{\text {cc }}$ of the MSP430 as follows:

$$
E_{P_{\mathrm{dec}}}^{(t)}(n)= \begin{cases}36\left(2 n t_{c}+2 t_{c}^{2}\right) \cdot m E_{\mathrm{cc}}, & n \geq L, \\ 0, & n=\{0, L\} .\end{cases}
$$

When the codeword length $n=\{0, L\}$, the node does not carry out any coding and (i.e., no redundancy overhead is added $L=n$ or no transmission takes place $n=0$.

4.2.3. Circuitry Energy. The static circuitry power consumption at the transmitter node is the sum of the power consumption for the block diagrams including the Digital-toAnalog Converter (DAC), the active filter (AF) at the transmitter side, and the frequency synthesizer (FS). Similarly, the circuit energy consumption at the receiver node is the sum of the power consumption values for the Low Noise Amplifier (LNA), the Intermediate Frequency Amplifier (IFA), the AF, the Analog-to-Digital Converter (ADC), and the FS. Thus, the circuitry power consumed by the transceiver is expressed as

$$
P_{c}=P_{\mathrm{DAC}}+2 P_{\mathrm{AF}}+2 P_{\mathrm{FS}}+P_{\mathrm{LNA}}+P_{\mathrm{IFA}}+P_{\mathrm{ADC}} \text {. }
$$

The circuitry energy of the transceiver given the power consumption $P_{c}$ and a period of time $T$ is given as follows:

$$
E_{c}=P_{C} \cdot T
$$

4.3. Energy Efficiency of JLSCC. In multihop WSN, sensor nodes sense and transmit their own readings as well as they process and forward their attached node data according to the multihop algorithm. In this work, we consider that sensed data is compressed at source nodes, while data reconstruction and decompression are carried out at the BS. In return, channel encoding and decoding are performed at source nodes and relay nodes (e.g., intermediate nodes and cluster heads), respectively, until the data is correctly received by the BS. In this case, the total energy cost or gain when using a lossy source-channel coding system with a particular temporal compression scheme and channel encoder/decoder implementation, at a given frequency, distance, throughput, and required $\mathrm{BER}$, can be expressed as the combination of its energy savings due to compression and coding gain, plus the energy cost due to compressor and encoder/decoder power consumptions. However, a lossy source-channel coding system is deemed to be energy efficient when the overall transmission energy saving due to compression and coding is strictly smaller than the cost associated with data processing at both transmitter and receiver.

Let $E_{t x}$ and $\widehat{E}_{t x}$ the energies of transmitting uncompresseduncoded packet and compressed-coded packet, respectively. The transmission energy gain $\Delta E_{S_{t x}}$ of transmitting a compressed and coded packet $\widehat{L}$ with respect to an uncompressed and uncoded packet $L_{0}$ is expressed as

$$
\Delta E_{S_{t x}}^{(t)}=\left(E_{t x}-\widehat{E}_{t x}\right) \geq 0 .
$$

A lossy source-coding system is considered to be energy efficient if and only if the total transmission energy saving $\Delta E_{S_{t x}}$ is larger than the total processing energy $E_{\text {proc }}$. The percentage of the energy efficiency $\theta$ is approximated as

$$
\theta^{(t)}=\frac{\Delta E_{S_{t x}}^{(t)}}{E_{\text {proc }}} \times 100
$$

where $E_{\text {proc }}=E_{\text {comp }}+E_{\text {dec }}$.

The transmission energy saving of channel coding depends mainly on the coding gain $C_{\text {gain }}$ of the used coding scheme and thus on the SNR of uncoded $\gamma_{U}$ and coded $\gamma_{\text {ECC }}$ packets. Accordingly, the SNR $\gamma_{U}$ of compressed, yet uncoded packet can be expressed as a function of the compression ratio. The packet error rate of a compressed packet given a certain SNR and compression ratio is given as

$$
\epsilon_{L}(\gamma, \rho)=1-\left(1-\epsilon_{b}(\gamma)\right)^{L(\rho)}
$$

where $L(\rho)$ is the compressed packet size expressed in bits and $\epsilon_{b}(\gamma)$ is the BER formula. The packet size depends on the compression ratio in the current time slot, i.e., $L(\rho)=\rho \cdot L_{0}$.

The SNR $\gamma_{U}$ of uncoded data is determined according to the selected modulation. Assuming an Offset Quadrature Phase Shift Keying (OQPSK) modulation, the BER is approximated as

$$
\epsilon_{b}(\gamma)=\frac{1}{2} \operatorname{erfc}(\sqrt{ } \gamma)
$$


where erfc is the complementary error function. From Equation (29), the SNR threshold $\gamma_{U}$ corresponding to the target packet error probability is given by

$$
\gamma_{U}=\left(\operatorname{erfc}^{-1}\left(2\left(1-\left(1-\epsilon_{L}\right)^{1 / L(\rho)}\right)\right)\right)^{2}
$$

The SNR $\gamma_{\text {ECC }}$ of compressed and coded packet can be expressed as a function of the coding rate and error probability of the implemented ECC coding scheme. Leveraging on [49], the SNR $\gamma_{\mathrm{ECC}}$ of OQPSK modulated and coded packet is expressed as

$$
\gamma_{\mathrm{ECC}}=\frac{1}{r} \frac{\operatorname{erfc}^{-1}\left(2 \epsilon_{s}\right)}{2(\sin \pi / 4)^{2}},
$$

where $\epsilon_{s}$ is the channel symbol error probability corresponding to the desired coded symbol error probability $\epsilon_{c s}$ of the implemented ECC coding scheme. For RS coding schemes, $\epsilon_{s}$ is approximated as

$$
\epsilon_{s}(R S)=\frac{1}{n} \sum_{i=t+1}^{n}\left(\begin{array}{l}
k \\
n
\end{array}\right) i\left(\epsilon_{c s}\right)^{i}\left(1-\epsilon_{c s}\right)^{n-1} .
$$

From Equations (30) and (31), the coding gain of coded information can be expressed as

$$
\begin{aligned}
C_{\text {gain }}= & \gamma_{U}-\gamma_{\mathrm{ECC}}=\left(\operatorname{erfc}^{-1}\left(2\left(1-\left(1-\epsilon_{L}\right)^{1 / L(\rho)}\right)\right)\right)^{2} \\
& -\frac{1}{r} \frac{\operatorname{erfc}^{-1}\left(2 \epsilon_{s}\right)}{2(\sin \pi / 4)^{2}} .
\end{aligned}
$$

The transmission energy saving due to channel coding stems from the achieved coding gain $\left(\Delta E_{S_{t x}}^{(t)} \approx C_{\text {gain }}\right)$. However, the extra energy costs of coding redundancies may restrain the energy saving. Therefore, the coding gain of channel coding scheme results in transmission energy saving if it matches the following condition:

$$
C_{\text {gain }} \geq 10 \log \left(\frac{1}{r}\right) \text {. }
$$

\section{Results and Discussion}

In this section, we evaluate the energy efficiency of the joint lossy source-channel coding compared to lossy compression and channel coding systems for energy-constrained sensor networking. In this context, we show how the source and channel coding parameters affects the energy efficiency and data reliability in end-to-end and multihop scenarios.

In this work, the transmission parameters are taken from the datasheet of a real device, namely, RC2400HP RF Transceiver Module (an RF module based on ZigBee and IEEE 802.15.4). This model used a central frequency and bandwidth of $2.441 \mathrm{GHz}$ and $B=5 \mathrm{MHz}$, respectively, while $\eta_{A}$ is computed to be $\left(\eta_{A}=0.23\right)$. The channel gains are computed using the standard path-loss model with a path-loss expo- nent equal to 3.5 (e.g., urban scenario) and are affected by Rayleigh fading. The overall noise power spectral density is $-167 \mathrm{dBm} / \mathrm{Hz}$, and the slot duration $T$ is determined as $T=n \cdot T_{b}$, with $T_{b}=1 \mu$ s given an OQPSK modulation. The parameters of the distortion curves are $a_{i}=0.35, b_{i}=$ 19.9, which have been derived empirically fitting the realistic rate-distortion curves. The energies associated with circuitry parameters are $P_{\mathrm{ADC}}=4.1 \mathrm{~mW}, P_{\mathrm{DAC}}=55 \mathrm{~mW}, P_{\mathrm{FS}}=13.7$ $\mathrm{mW}, P_{\mathrm{AF}}=6.49 \mathrm{~mW}, P_{\mathrm{LNA}}=0.55 \mathrm{~mW}$, and $P_{\mathrm{IFA}}=0.2 \mathrm{~mW}$. The transmission energy at the current time slot is computed according to the adopted compression ratios $(\rho \in\{0,1\})$, the coding rates $(\rho \in\{0,1\})$, the required $\left(\mathrm{BER} \cong 10^{-4}\right)$, the transmission distance, the channel gains, and the original packet size $\left(L_{0}=50\right.$ bits $)$. Besides, sensor nodes can decide among 10 compression ratios and coding rates according to 10 levels of compression and coding.

5.1. Energy Analysis of JLSCC. In Figure 2, we plot the transmission energy consumed during a time slot $t$ in function of both compression ratio (Figure $2(\mathrm{a})$ ) and coding rate (Figure 2(b)) with a lossy compression, a channel coding, and JLSCC systems. In the first case (Figure 2(a)), the simulation results show that the transmission energy of a compressed packet with lossy compression decreases linearly with decreasing the compression ratio (i.e., reducing the packet size). When using the JLSCC system, the transmission energy is affected according to the used coding rate. Therein, three levels of coding rates $r \in\{0.3,0.5,0.8\}$ (i.e., high, medium, and low) are selected to be used in the JLSCC system, respectively. The JLSCC system may result in better performance than lossy compression as it benefits from both energy saving due to compression and coding gains. However, the quantity of channel coding redundancy can restrain the efficiency of the JLSCC system, yet enhances data reliability. For instance, using a JLSCC system with a coding rate of $(r=0.8)$ allows to outperform the lossy compression performance for all compression levels. This is because of the additional gain stemmed from the channel coding scheme that is not impacted by the small overhead of coding redundancy. In the other case, when the JLSCC system relies on higher levels of redundancy by using lower coding rates $r \in\{0.5$, $0.3\}$, the transmission energy remarkably increases and may even exceed the uncompressed and uncoded system when using compression ratios $\rho \in[0.88,1]$ and $\rho \in[0.65,1]$, respectively. In this case, the extra cost of the coding redundancy outweighs the energy saved by both compression and coding gains.

In the second case (Figure 2(b)), we assess the transmission energy of a channel coding system instead of lossy compression system, along with JLSCC systems, for various channel coding rates. The results show that when using the channel coding system, the transmission energy increases exponentially with decreasing the coding rates (i.e., increasing redundancies). However, it is noticed that when the coding rate is approximately less than $(r \leq 0.5)$, the channel coding system is energy efficient compared to the uncoded system. This refers to the energy saving of the coding gain $\left(S N R_{\mathrm{ECC}}<S N R_{U}\right)$ on the one hand and due to the fact that the extra energy of redundancy is less than the energy saving 


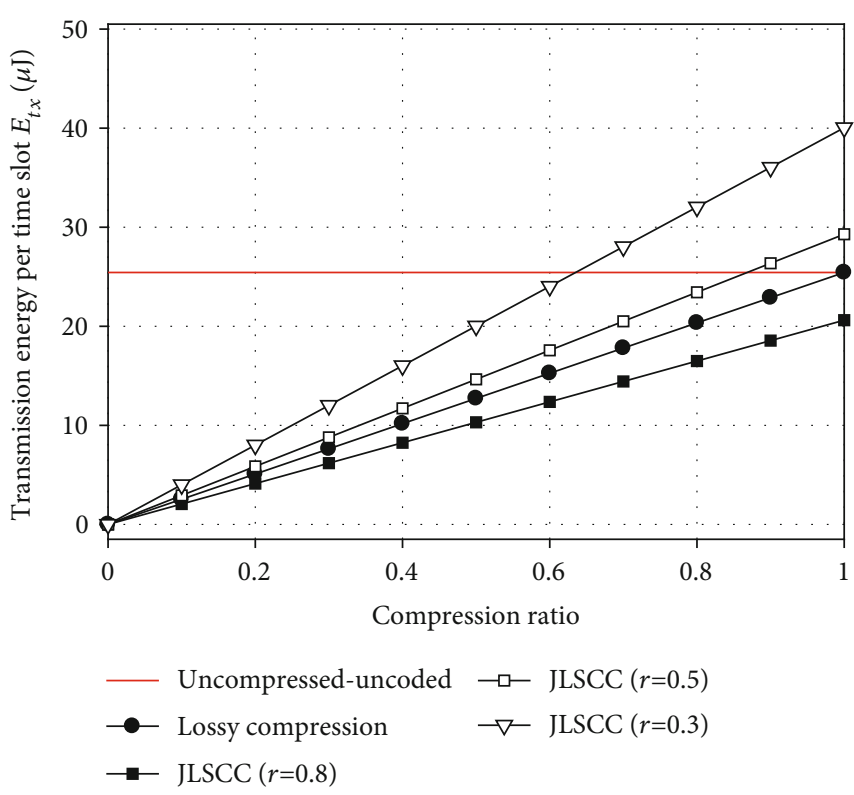

(a) Transmission energy vs. compression ratio

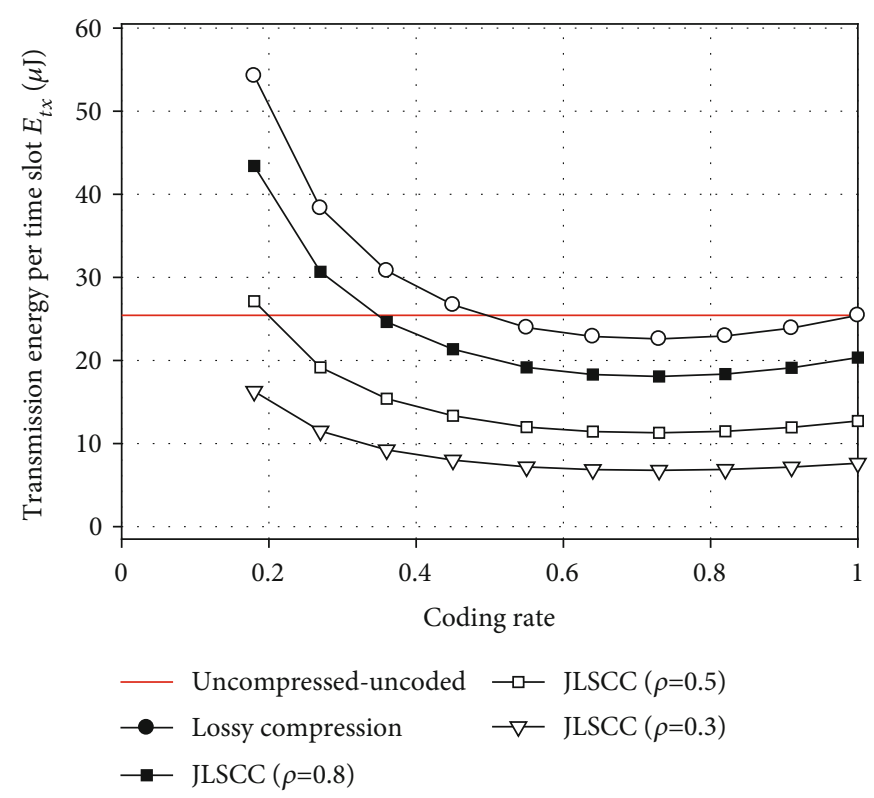

(b) Transmission energy vs. coding rate

Figure 2: Transmission energy consumption per time slot versus (a) compression ratio (b) coding rate.

on the other hand. When using JLSCC systems, the transmission energy per time slot performs efficiently compared to the channel coding system for various coding rate levels. In addition, the transmission energy efficiency of the JLSCC system increases by lowering the compression ratio from $\rho \in\{0.8,0.5,0.3\}$, respectively. This is explained by the fact that lowering the compression ratio brings about smaller channel codeword to be transmitted over the communication channel. Although reducing the compression ratio leads to increased source distortion, the data can be optimally reconstructed and decoded due to the channel coding system. It is however noticed that the JLSCC systems can be uneconomical at several coding rate levels, which is referred to the extra size of transmitted codeword settled by both compression and coding rates. In this case, both the codeword size and the transmission energy outweigh the original packet size and energy saving, respectively.

In sensor nodes, energy is affected by both transmission and processing energy. Accordingly, using lossy compression and channel coding schemes engenders the consideration of both extra computational energy of source compression and channel decoding at the transmitter and receiver, respectively. Although lossy compression and channel error correction allow to save transmission energy and provide reliability, their processing energy often restricts the energy saving and thus the energy efficiency of the source-channel coding system. In Figure 3, we plot both compression and channel decoding energy expenditure as function of compression ratio and codeword length, respectively. The processing energies are computed according to the low power MSP430F16 MCU. It can be noticed that the compression energy of the lossy compression scheme increases linearly with decreasing the compression ratio (i.e., compressing more the data). Consequently, the transmission energy saved by the compression may likely be constrained by the computational energy of compression. Similarly, the decoding energy of ECC grows with increasing the coding length $n$ (i.e., decreasing the coding rate) of the transmitted codewords, which inversely affects the transmission energy saving. However, the computational process of ECC decoding is more energy demanding than compression process. Therefore, both source and channel coding processing energies must not be neglected in sensor nodes at both transmitters and receivers. This is due to the main tradeoff between transmission and processing energies on the one hand and between communication reliability performance and energy saving on the other hand, when using a JLSCC system.

In Figure 4, the transmission energy saving of lossy compression and JLSCC systems is plotted in function of various compression ratios and several selected coding rates. In this case, we only consider the transmission energy consumption without including the processing energy burdens of compression and coding. The results exhibit that the energy saving of lossy compression system is linearly dependent on the compression ratio, so that the percentage of energy saving increases from $0 \%$ to $100 \%$ with decreasing the compression ratio from 1 to 0 . Accordingly, the lossy compression system is always energy efficient unless the data is not compressed $(\rho=1)$. When using a JLSCC system to overcome the channel impairments, sensor nodes are expected to gain extra energy saving due to channel coding gain. However, the quantity of redundancy may impact the energy saved by coding gain and compression. For instance, when using a coding rate of $(r=0.8)$, it can be noticed that the JLSCC system outperforms completely the lossy compression system at all compression levels by saving more energy, which can be deemed crucial for both energy and reliability requirements. In contrast, when using lower coding rates $(r=0.5,0.3)$, the JLSCC systems save less transmission energy than the lossy compression system and 

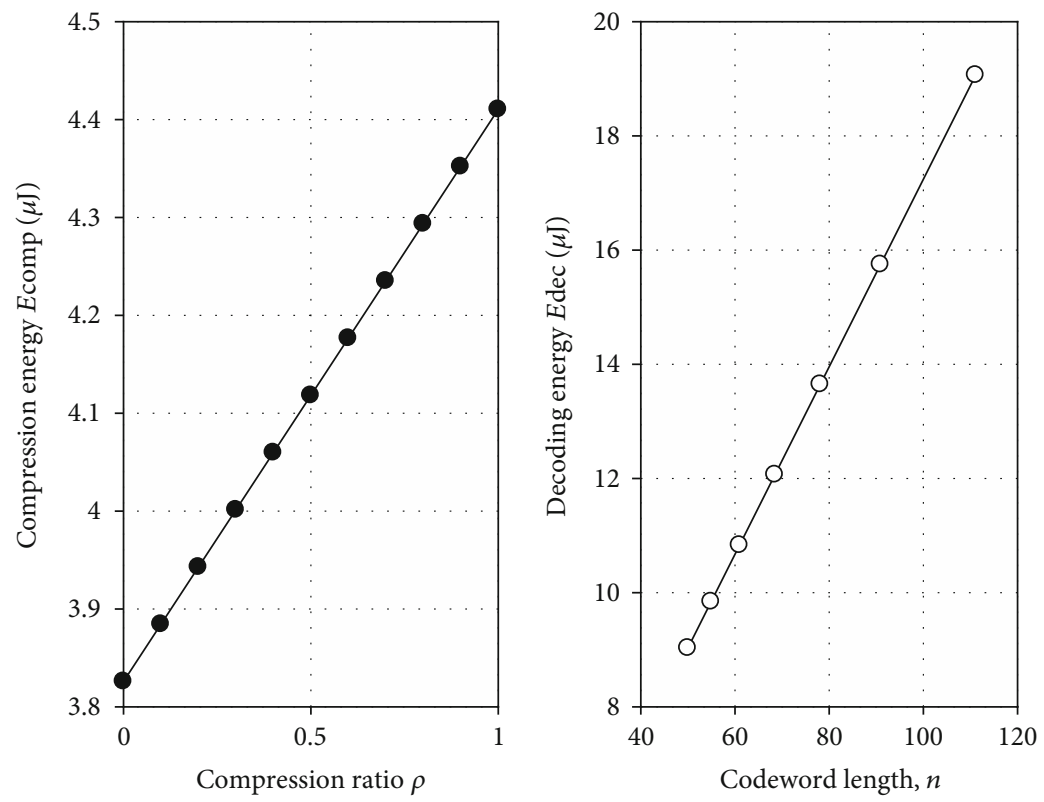

FIGURE 3: Processing energy of compression and channel decoding vs. compression ratio and coding length, respectively.

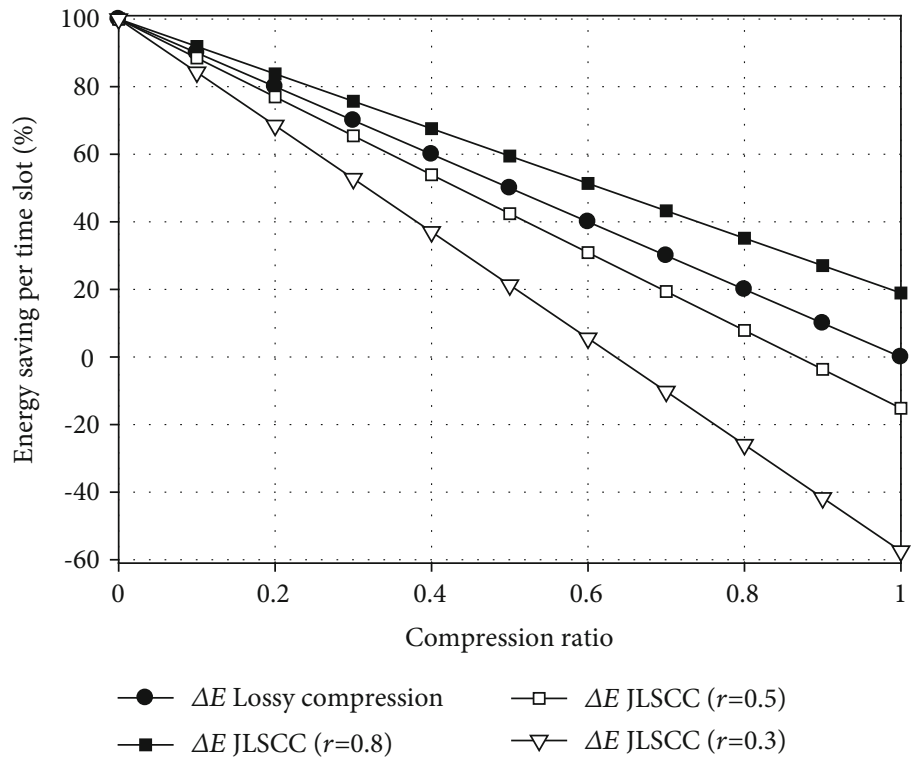

FIgURE 4: Transmission energy saving per time slot $\Delta E_{t x}^{(t)}$ versus compression ratio for a single hop scenario.

can even consume more energy than the uncoded scheme for several compression levels $\rho \in[0.87,1]$ and $\rho \in[0.62,1]$, respectively. This is explained by the sizable length of the transmitted codeword that can exceed the original packet size when using lower coding rates, in order to meet data fidelity requirements. Therefore, JLSCC systems can guarantee more transmission energy saving than lossy compression if a suitable allocation between source and channel coding is acquired.

In Figure 5, the energy efficiency of compression and JLSCC systems is shown as function of compression ratio considering two cases: computational energy of compression is considered at transmitter nodes, yet decoding is performed at the BS (Figure 5(a)); both computation costs of compression and decoding are counted at the transmitter and intermediate node (Figure 5(b)), respectively. Therein, both transmission and processing energies of lossy compression and coding systems are taken into account for the energy efficiency of sensor nodes. In this case, extra compression and channel decoding processing dissipation are considered at the transmitter and receiver nodes, respectively. Accordingly, nodes are expected to consume more energy with JLSCC systems, as providing energy efficiency requires that the transmission energy saving must exceed the related processing energy. To this end, we plot first the energy efficiency of lossy compression and JLSCC systems considering exclusively the 


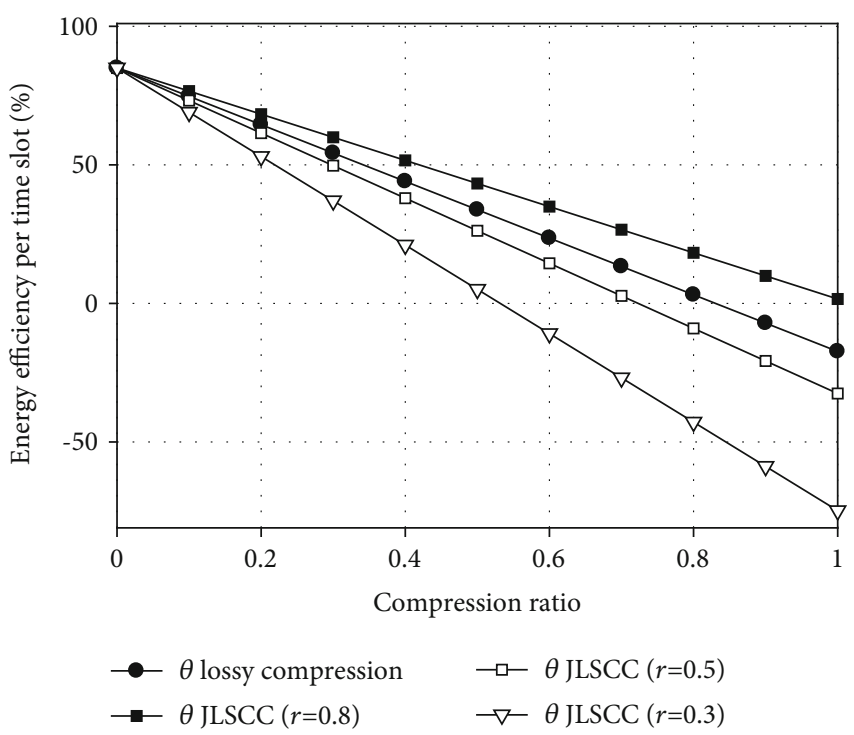

(a) Decoding at the BS

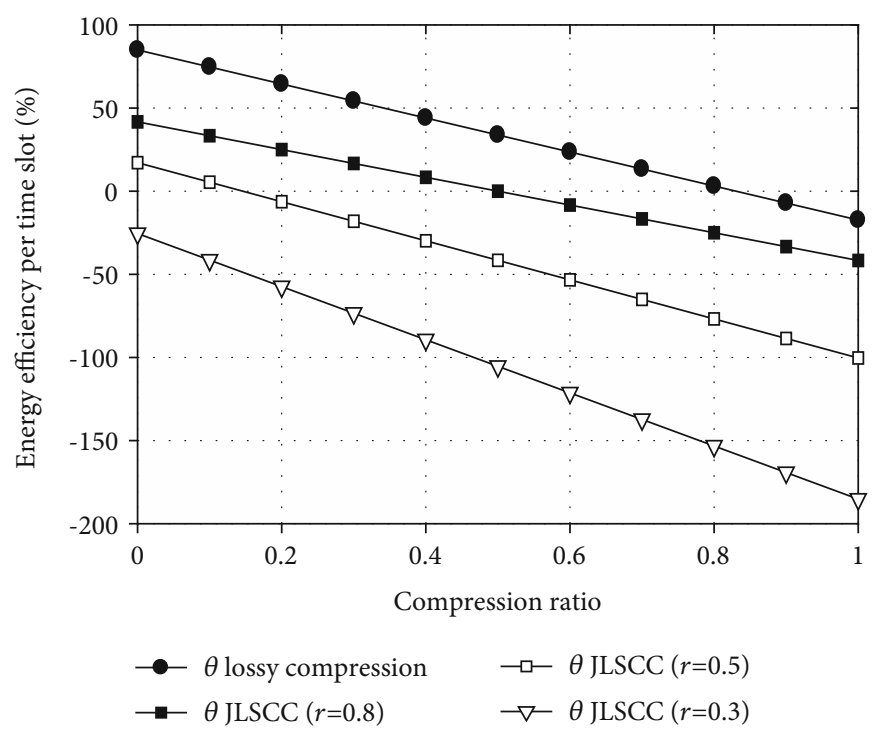

(b) Decoding at the receiver

FIgURE 5: Total energy efficiency versus compression ratio for single hop, when (a) decoding is carried out at the receiver which is the BS, (b) decoding is carried out at the receiver which is an intermediate node or cluster head. The computation energy of decoding is counted according to the used coding rate of JLSCC systems.

computational costs of compression in Figure 5(a). The results reveal that for both lossy compression and JLSCC systems, the energy efficiency is achieved for most compression ratios; yet if achieved, the percentage of energy saving decreases remarkably compared to Figure 4 when only communication energy is considered. For instance, in lossy compression system, the percentage of energy efficiency varies from 0 to $76 \%$, when the compression ratio varies from $\epsilon[0.77,0]$, respectively. In the other case, when $\rho \in[0.77,1]$, the lossy compression system becomes energy inefficient by consuming more energy than the uncompressed system. Similarly, by using the JLSCC systems, the energy efficiency decreases as much as we decrease the coding ratio. However, the JLSCC system with a coding rate of $(r=0.8)$ maintains the best performance in terms of energy efficiency at all compression levels in spite of including compression cost. Notably, the JLSCC systems turn out to drain more energy by lowering the coding rate from $=0.5$ to 0.3 . In the second scenario (Figure 5(b)), we plot the energy efficiency of communication systems taking into account the computational costs of both compression and error control decoding at the transmitter and receiver, respectively. In this case, the energy efficiency of JLSCC systems significantly decreased and even crossed the uncoded system energy threshold at certain compression levels, yet the JLSCC system with $(r=0.3)$ turns out to be completely energy inefficient. This can be justified by the inherent impact of the additional processing cost of error control decoding at the receiver that, plus the transmission energy, can exhaust the energy resources of the transceiver. Instead, the JLSCC system with $(r=0.8)$ can lead to satisfactory performance in terms of energy efficiency, in spite of the lightweight decrease for compression ratios $(\rho \geq 0.8)$. Consequently, the energy efficiency of the JLSCC system is solely dependent on the desired level of reliability expected by using the ECC scheme. Note that using lower coding rate (i.e., more redundancy) enables to provide enhanced reliability than using higher rates. This is, however, guaranteed at the cost of higher computational energy at both transmitter and receiver.

5.2. Multihop Performance of JLSCC. In multihop networks, nodes are likely going to process the received data before transmission in order to assure the required reconstruction and reliability performance. In this case, compressing the sensed data at source nodes as well as decoding the data at several intermediate nodes is extremely requisite in highly lossy environment. Therein, we assess the energy efficiency of the adaptive JLSCC systems in a multihop fashion.

In Figure 6, we focus on multihop communications and evaluate whether further gains are possible when the compressed and coded information has to travel multiple hops to reach the base station. In this case, both transmission and reception energies are counted at each intermediate node in the network. In Figure 6(a), we neglect the processing energy of decoding at intermediate nodes by assuming that decoding is carried out at the BS, and we assess the possible energy gains of both lossy compression and JLSCC systems. We set the compression ratio $\rho=0.5$ and the coding rate on $r \in\{0.3,0.5,0.8\}$. As shown in this figure, the energy efficiency of the network increases with the number of hopes for both lossy compression and JLSCC systems. This is due to the fact that, although the energy spent for the compression and coding at the source nodes is comparable to that spent for the transmission, these computational energies are only considered at source nodes, while other relay nodes are only forwarding the received data. This leads to extra energy gains (i.e., of compression and ECC), which are accumulated with the number of transmission hopes. In the other case, we 


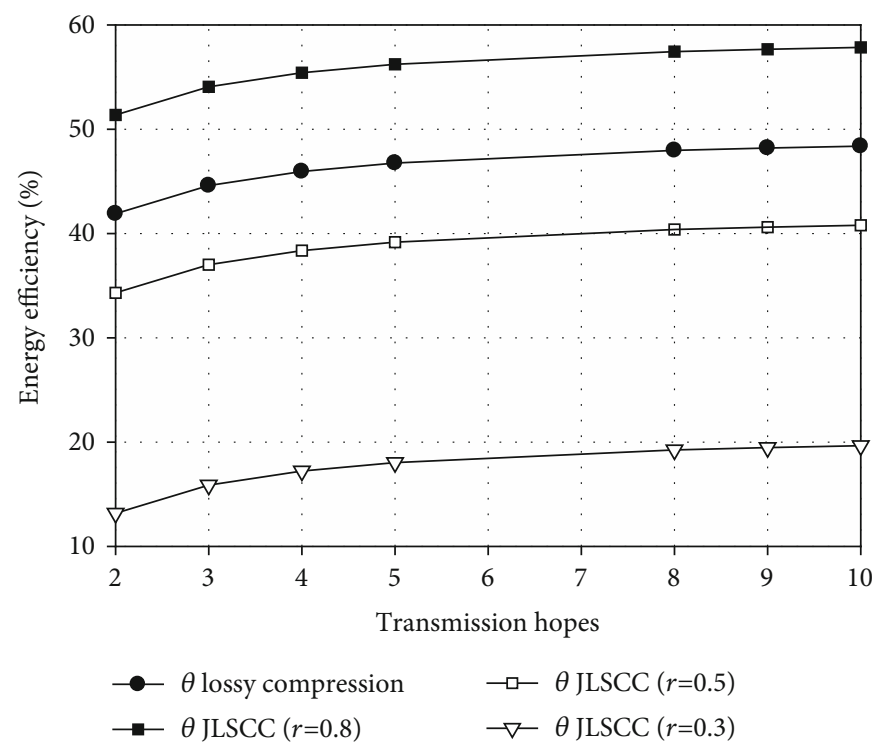

(a) Decoding at the BS

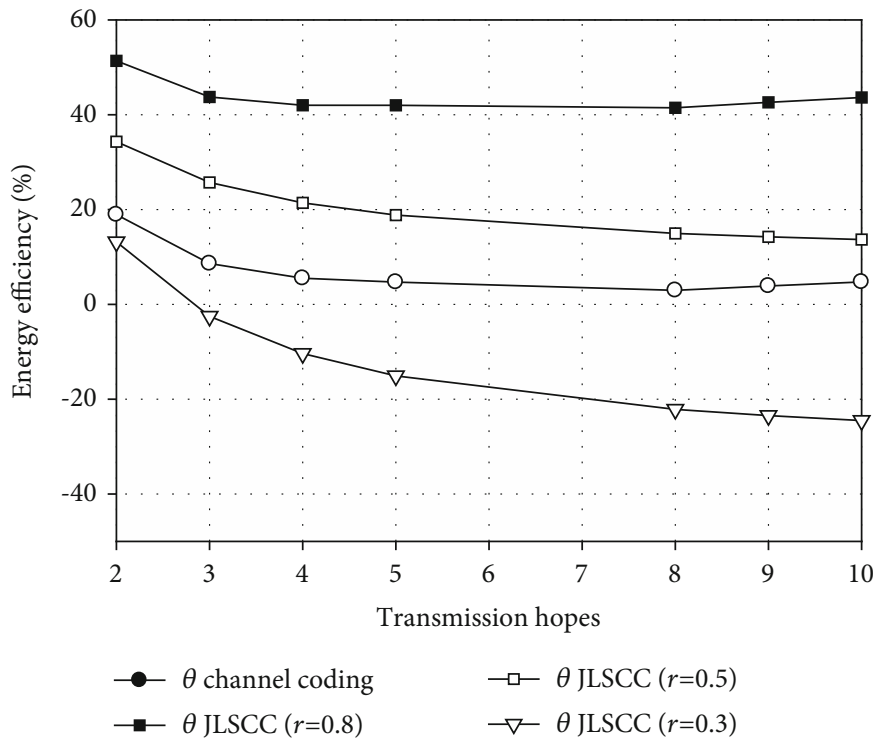

(b) Decoding at $50 \%$ of nodes

FIGURE 6: Energy efficiency versus transmission hopes for multihop scenario, when (a) computational energy of compression is considered at transmitter nodes, yet decoding is carried out at the receiver, which is the BS, (b) both computation costs of compression and decoding are counted at the transmitters and intermediate nodes, respectively.

evaluate the energy efficiency when several nodes in the network are expected to process the data before transmission (e.g., relay nodes and cluster heads). Therein, the decoding energy is considered for the elected relay nodes in the network. In Figure 6(b), 50\% of nodes in the network are assumed to carry out decoding before forwarding data to the BS. The results show that the energy efficiency of JLSCC systems decreases with the number of hopes, especially with a coding rate of $(r=0.3)$ that requires higher computational energy. This is because of the additional cost of the decoding at intermediate nodes, that is, plus the compression energy, allows to impact extensively the energy saved in transmission. However, it is noticed that in spite of the lightweight energy efficiency decrease of JLSCC systems with $(r=$
$0.5,0.8)$, that is, traded off with enhanced data reliability, they can also guarantee more than $10 \%$ and $40 \%$ of energy efficiency, respectively. We note that when using higher redundancy in the JLSCC systems, the processing energy of ECC increases, which leads to reduce the energy efficiency at the cost of more data reliability.

In Figure 7, we show the energy efficiency versus the distance between hops of lossy compression (LC) and JLSCC systems. Given the distance, the transmission power is selected according to the Friis path-loss formula (with pathloss exponent $\alpha=3.5$ ). For each value of the distance, we evaluated the energy efficiency using the minimum transmission power level that leads to the required BER for each coding rate. As shown in Figure 7, the energy efficiency 


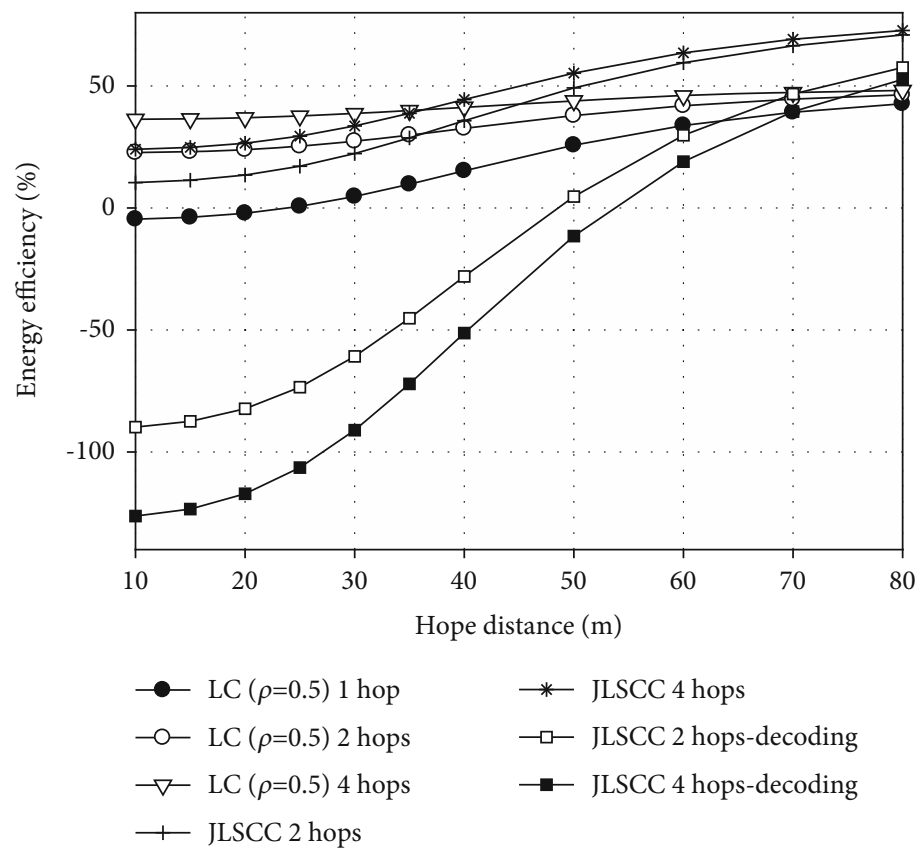

FIGURE 7: Energy efficiency versus hop distance for LC and JLSCC systems ( $\rho=0.5, r=0.5)$.

increases remarkably with the distance for both LC and JLSCC systems, even when considering both computational costs of compression and ECC. This is due to the fact that the transmission energy becomes progressively higher than that needed for compression and ECC processing. This effect becomes more pronounced when the number of hops is increased, as the relay nodes are benefiting from higher energy gains (i.e., correspond to compression and coding gains). Accordingly, when the transmission distances are long, the adaptive JLSCC system can guarantee both energy efficiency and high data reliability, by relying on compression and coding for both transmitters and intermediate nodes.

\section{Conclusion}

In this work, we have systematically analyzed the performance of joint lossy source-channel coding for constrained sensor networking by investigating whether both energy efficiency and reliability are possible, depending on lossy compression and channel coding performance and hardware characteristics. Both the communication and the processing costs have been taken into account in end-to-end and multihop architectures. Our results reveal that the consideration of JLSCC in end-to-end transmission scenario, where compression is carried out at the transmitter, and ECC decoding at the BS, results in substantial energy efficiency compared to lossy compression while maintaining the required reliability. However, the computational energy of compression at the transmitters and decoding at intermediate nodes can outweigh that spent for transmission at the physical layer, in multihop wireless communication. In this context, using JLSCC can lead to significant energy efficiency when the channel decoding is only performed at the BS, by taking advantage of both compression and coding gains. However, when decoding is executed at several intermediate nodes or cluster heads in order to guarantee more reliability, the energy efficiency is impacted by the computation cost, especially in small transmission distances, yet can lead to satisfactory energy saving in large transmission distances.

\section{Data Availability}

The simulation parameter data used to support the findings of this study are included within the article.

\section{Conflicts of Interest}

The authors declare that there is no conflict of interest regarding the publication of this paper.

\section{References}

[1] A. H. A. Fahmy, "Wireless sensor network applications," in Wireless Sensor Networks, R. A. Lewin, Ed., pp. 69-213, Springer, Singapore, 2016.

[2] L. Huo and D. Jiang, "Stackelberg game-based energy-efficient resource allocation for $5 \mathrm{G}$ cellular networks," Telecommunication Systems, vol. 72, no. 3, pp. 377-388, 2019.

[3] N. S. Alhassoun, M. Y. Sarwar Uddin, and N. Venkatasubramanian, "Context-aware energy optimization for perpetual IoT-based safe communities," Sustainable Computing: Informatics and Systems, vol. 22, pp. 96-106, 2019.

[4] S. Yadav and R. S. Yadav, "A review on energy efficient protocols in wireless sensor networks," Wireless Networks, vol. 22, no. 1, pp. 335-350, 2016.

[5] M. Hammoudeh, F. al-Fayez, H. Lloyd et al., "A wireless sensor network border monitoring system: deployment issues and routing protocols," IEEE Sensors Journal, vol. 17, no. 8, pp. 2572-2582, 2017.

[6] D. Jiang, W. Li, and H. Lv, "An energy-efficient cooperative multicast routing in multi-hop wireless networks for smart 
medical applications," Neurocomputing, vol. 220, pp. 160-169, 2017.

[7] D. Zordan, B. Martinez, I. Vilajosana, and M. Rossi, "On the performance of lossy compression schemes for energy constrained sensor networking," ACM Transactions on Sensor Networks, vol. 11, no. 1, pp. 1-34, 2014.

[8] G. Quer, R. Masiero, G. Pillonetto, M. Rossi, and M. Zorzi, "Sensing, compression, and recovery for WSNs: sparse signal modeling and monitoring framework," IEEE Transactions on Wireless Communications, vol. 11, no. 10, pp. 3447-3461, 2012.

[9] E. Fasolo, M. Rossi, J. Widmer, and M. Zorzi, "In-network aggregation techniques for wireless sensor networks: a survey," IEEE Wireless Communications, vol. 14, no. 2, pp. 7087, 2007.

[10] D. Jiang, W. Wang, L. Shi, and H. Song, "A compressive sensing-based approach to end-to-end network traffic reconstruction," IEEE Transactions on Network Science and Engineering, vol. 7, no. 1, pp. 507-519, 2020.

[11] I. Ez-zazi, M. Arioua, A. el Oualkadi, and P. Lorenz, “A hybrid adaptive coding and decoding scheme for multi-hop wireless sensor Networks," Wireless Personal Communications, vol. 94, no. 4, pp. 3017-3033, 2017.

[12] M. E. Pellenz, R. D. Souza, and M. S. P. Fonseca, "Error control coding in wireless sensor networks," Telecommunication Systems, vol. 44, no. 1-2, pp. 61-68, 2010.

[13] S. L. Howard, C. Schlegel, K. Iniewski, and K. Iniewski, "Error control coding in low-power wireless sensor networks: when is ECC energy-efficient?," EURASIP Journal on Wireless Communications and Networking, vol. 2006, no. 1, Article ID 074812, 2006.

[14] R. Sukjaimuk, Q. Nguyen, and T. Sato, “A smart congestion control mechanism for the green IoT sensor-enabled informationcentric networking," Sensors, vol. 18, no. 9, p. 2889, 2018.

[15] J. Č. Gambiroža and T. Mastelic, "Big data challenges and trade-offs in energy efficient internet of things systems," in 2018 26th International Conference on Software, Telecommunications and Computer Networks (SoftCOM), pp. 1-6, Split, Croatia, September 2018.

[16] G. Anastasi, M. Conti, M. Di Francesco, and A. Passarella, "Energy conservation in wireless sensor networks: a survey," Ad Hoc Networks, vol. 7, no. 3, pp. 537-568, 2009.

[17] S. Ulukus, A. Yener, E. Erkip et al., "Energy harvesting wireless communications: a review of recent advances," IEEE Journal on Selected Areas in Communications, vol. 33, no. 3, pp. 360381, 2015.

[18] D. Jiang, P. Zhang, Z. Lv, and H. Song, "Energy-efficient multiconstraint routing algorithm with load balancing for smart city applications," IEEE Internet of Things Journal, vol. 3, no. 6, pp. 1437-1447, 2016.

[19] F. Wang, D. Jiang, and S. Qi, “An adaptive routing algorithm for integrated information networks," China Communications, vol. 16, no. 7, pp. 195-206, 2019.

[20] V. L. Quintero, C. Estevez, M. E. Orchard, and A. Pérez, "Improvements of energy-efficient techniques in WSNs: a MAC-protocol approach," IEEE Communications Surveys Tutorials, vol. 21, no. 2, pp. 1188-1208, 2019.

[21] A. Biason, C. Pielli, A. Zanella, and M. Zorzi, "Access control for IoT nodes with energy and fidelity constraints," IEEE Transactions on Wireless Communications, vol. 17, no. 5, pp. 3242-3257, 2018.
[22] M. C. Vuran and I. F. Akyildiz, "Error control in wireless sensor networks: a cross layer analysis," IEEE/ACM Transactions on Networking, vol. 17, no. 4, pp. 1186-1199, 2009.

[23] D. Incebacak, R. Zilan, B. Tavli, J. M. Barcelo-Ordinas, and J. Garcia-Vidal, "Optimal data compression for lifetime maximization in wireless sensor networks operating in stealth mode," Ad Hoc Networks, vol. 24, pp. 134-147, 2015.

[24] L. D. P. Mendes and J. J. P. C. Rodrigues, "A survey on crosslayer solutions for wireless sensor networks," Journal of Network and Computer Applications, vol. 34, no. 2, pp. 523-534, 2011.

[25] M. A. Razzaque, C. Bleakley, and S. Dobson, "Compression in wireless sensor networks," ACM Transactions on Sensor Networks, vol. 10, no. 1, pp. 1-44, 2013.

[26] D. del Testa and M. Rossi, "Lightweight lossy compression of biometric patterns via denoising autoencoders," IEEE Signal Processing Letters, vol. 22, no. 12, pp. 2304-2308, 2015.

[27] A. Guitton, A. Skordylis, and N. Trigoni, "Utilizing correlations to compress time-series in traffic monitoring sensor networks," in 2007 IEEE Wireless Communications and Networking Conference, pp. 2479-2483, Kowloon, China, March 2007.

[28] T. Schoellhammer, B. Greenstein, E. Osterweil, M. Wimbrow, and D. Estrin, "Lightweight temporal compression of microclimate datasets [wireless sensor networks]," in 29th Annual IEEE International Conference on Local Computer Networks, pp. 516-524, Tampa, FL, USA, November 2004.

[29] M. A. Mahmood, W. K. G. Seah, and I. Welch, "Reliability in wireless sensor networks: a survey and challenges ahead," Computer Networks, vol. 79, pp. 166-187, 2015.

[30] A. Aminjavaheri, A. RezazadehReyhani, R. Khalona, H. Moradi, and B. Farhang-Boroujeny, "Underlay control signaling for ultra-reliable low-latency IoT communications," in 2018 IEEE International Conference on Communications Workshops (ICC Workshops), pp. 1-6, Kansas City, MO, USA, May 2018.

[31] M. S. Motlagh, M. B. Khuzani, and P. Mitran, "On lossy joint source-channel coding in energy harvesting communication systems," IEEE Transactions on Communications, vol. 63, no. 11, pp. 4433-4447, 2015.

[32] P. Castiglione, O. Simeone, E. Erkip, and T. Zemen, "Energy management policies for energy-neutral source-channel coding," IEEE Transactions on Communications, vol. 60, no. 9, pp. 2668-2678, 2012.

[33] O. Orhan, D. Gündüz, and E. Erkip, "Source-channel coding under energy, delay, and buffer constraints," IEEE Transactions on Wireless Communications, vol. 14, no. 7, pp. 38363849, 2015.

[34] K. Zachariadis, M. Honig, and A. Katsaggelos, "Source fidelity over fading channels: performance of erasure and scalable codes," IEEE Transactions on Communications, vol. 56, no. 7, pp. 1080-1091, 2008.

[35] I. E. Aguerri and D. Gunduz, "Expected distortion with fading channel and side information quality," in 2011 IEEE International Conference on Communications (ICC), pp. 1-6, Kyoto, Japan, June 2011.

[36] I. Ez-zazi, M. Arioua, and A. el Oualkadi, "Analysis of lossy compression and channel coding tradeoff for energy efficient transmission in low power communication systems," in 2018 9th International Symposium on Signal, Image, Video and Communications (ISIVC)no. p, pp. 291-295, Rabat, Morocco, November 2018. 
[37] C. Pielli, Č. Stefanović, P. Popovski, and M. Zorzi, "Joint compression, channel coding, and retransmission for data fidelity with energy harvesting," IEEE Transactions on Communications, vol. 66, no. 4, pp. 1425-1439, 2018.

[38] I. Ez-zazi, M. Arioua, A. El Oualkadi, and P. Lorenz, “On the performance of adaptive coding schemes for energy efficient and reliable clustered wireless sensor networks," Ad Hoc Networks, vol. 64, pp. 99-111, 2017.

[39] J. H. Kleinschmidt, "Analyzing and improving the energy efficiency of IEEE 802.15.4 wireless sensor networks using retransmissions and custom coding," Telecommunication Systems, vol. 53, no. 2, pp. 239-245, 2013.

[40] I. Ez-zazi, A. Eloualkadi, M. Arioua, and Y. Elassari, "Performance analysis of efficient coding schemes for wireless sensor networks," in Proceedings of IEEE international Workshop on RFID and Wireless Sensor Network (RAWSN'15), pp. 42-47, Morocco, May 2015.

[41] I. Ez-zazi, M. Arioua, and A. El Oualkadi, "On the design of coding framework for energy efficient and reliable multi-hop sensor networks," Procedia Computer Science, vol. 109, pp. 537-544, 2017.

[42] M. Y. Naderi, H. R. Rabiee, M. Khansari, and M. Salehi, "Error control for multimedia communications in wireless sensor networks: a comparative performance analysis," Ad Hoc Networks, vol. 10, no. 6, pp. 1028-1042, 2012.

[43] Y. Polyanskiy, H. V. Poor, and S. Verdu, "Channel coding rate in the finite blocklength regime," IEEE Transactions on Information Theory, vol. 56, no. 5, pp. 2307-2359, 2010.

[44] W. Yang, G. Durisi, T. Koch, and Y. Polyanskiy, "Quasi-static Multiple-Antenna fading channels at finite blocklength," IEEE Transactions on Information Theory, vol. 60, no. 7, pp. 42324265, 2014.

[45] T. S. Rappaport, Wireless Communications: Principles and Practice, Prentice Hall, Reading, MA, 1996.

[46] C. Karakus, A. C. Gurbuz, and B. Tavli, "Analysis of energy efficiency of compressive sensing in wireless sensor networks," IEEE Sensors Journal, vol. 13, no. 5, pp. 1999-2008, 2013.

[47] L. Bierl, MSP430 family mixed-signal microcontroller application reports, Tech. Rep. SLAA024: Texas Instruments Incorporated, Dallas, TX, USA, 2000.

[48] J. Singh and D. Pesch, "Application of energy efficient softdecision error control in wireless sensor networks," Telecommunication Systems, vol. 52, no. 4, pp. 2573-2583, 2013.

[49] J. G. Proakis, Digital Communications, McGrawHill, New York, USA, 4th edition, 2001. 